\title{
Fat Necrosis in the Post-Surgical Breast: Dependence on Adjuvant Treatment and Morphology on Digital Mammography
}

\author{
Heike Preibsch ${ }^{1,{ }^{*}}$ and Matthew G Wallis ${ }^{2}$ \\ ${ }^{1}$ Diagnostic and Interventional Radiology, University Hospital Tübingen, Tubingen, Germany \\ ${ }^{2}$ Cambridge Breast Unit, NIHR Cambridge Biomedical Research Centre, Cambridge University Hospitals NHS Foundation Trust, Cambridge Biomedical Campus, Cambridge, \\ United Kingdom \\ "Corresponding author: Diagnostic and Interventional Radiology, University Hospital Tübingen, Hoppe-Seyler-St. 3, 72076 Tübingen, Germany, Fax: +49-7071295499, Email: \\ heike.preibsch@med.uni-tuebingen.de
}

Received 2019 February 14; Revised 2019 July 28; Accepted 2019 October 12.

\begin{abstract}
Background: Fat necrosis is a common finding after trauma or surgery of the breast. There are various presentations on imaging and differentiation from malignancy can be challenging.

Objectives: To analyze early signs of fat necrosis on postoperative mammograms after the excision of benign and malignant findings in accordance to modern adjuvant treatment.

Patients and Methods: A retrospective analysis was performed as part of an audit. Mammograms of 39 patients, who underwent surgical excision of benign disease, and 81 patients with cancer surgery, were reviewed. Adjuvant therapy, such as radiotherapy, chemotherapy and hormonal treatment, was noted.

Results: Mean patient age was $54.6 \pm 6.5$ years in the benign and $59.8 \pm 7.8$ years in the malignant group. Mean lesion size did not differ between the groups $(\mathrm{P}=0.735)$. Development of fat necrosis on mammography follow-up was observed in $12.8 \%(5 / 39)$ of the patients in the benign and in 32.1\% (26/81) of the patients in the malignant group ( $\mathrm{P}=0.024)$. Mean follow-up time until the first sign of fat necrosis was 2.6 years in the benign group and 2.0 years in the malignant group $(\mathrm{P}=0.187)$. With radiation therapy \pm hormonal treatment 25 of 75 patients (33.3\%) developed fat necrosis. Hormonal treatment significantly influenced the development of fat necrosis in the malignant group (odds ratio [OR]: 0.231; $\mathrm{P}=0.029$ ). Four of 16 patients $(25.0 \%)$ having radiation and chemotherapy developed fat necrosis.

Conclusion: Fat necrosis development was observed significantly more often in patients after breast cancer surgery and modern adjuvant treatment compared to benign breast surgery. However, hormonal adjuvant treatment seemed to lower the chance of fat necrosis development.
\end{abstract}

Keywords: Breast, Mammography, Fat Necrosis, Adjuvant Treatment, Follow-Up

\section{Background}

Fat necrosis is a common finding in the post-surgical or post-traumatic breast $(1,2)$. It is a nonsuppurative inflammatory change of adipose tissue. On imaging, fat necrosis shows a wide variety of presentations (3-5). Mammographically, it could manifest as lipid cysts, coarse calcifications, focal asymmetries, microcalcifications, radiolucent or even spiculated masses $(6,7)$. The most common findings are dystrophic calcifications and radiolucent oil cysts (8). About one third of fat necrosis appears as round or oval radiolucent oil cyst with thin capsule and egg-shell calcifications (9). Especially in case of early calcification of the fibrous rim of an oil cyst or pure calcifications at the site of previous malignancy, discrimination between early fat necrosis and cancer recurrence could be challenging (10,
11). Fat necrosis shows pleomorphic calcifications suspicious for malignancy in $4 \%$ of the cases $(4,6,7)$.

Clinically, fat necrosis often manifests as a hard, palpable lump, and as it often occurs around the tumour bed this can cause anxiety in patients. Therefore, imaging to exclude recurrent disease is of great importance.

It is known that iatrogenic or non-iatrogenic trauma to the breast can induce the development of fat necrosis $(1,2$, $12,13)$. The mean time between breast trauma and the clinical manifestation of fat necrosis as a palpable lump is 68.5 weeks (2). In the absence of a history of trauma most fat necrosis lesions are found in the upper inner quadrant of the breast, which is thought to be due to the use of seatbelts (14).

Another known factor to increase the incidence of fat 
necrosis after breast conserving therapy is radiation therapy $(13,15)$. The 5-year rate of fat necrosis after breast conserving surgery and irradiation is reported to be up to $40 \%$ (16). A higher rate of fat necrosis after brachytherapy compared to whole breast irradiation was observed (16). The applicator size significantly influences the development of fat necrosis after intraoperative radiation (17). The formation of fat necrosis after radiation therapy without breast surgery is still unknown (15). In breast reconstruction, adjuvant chemotherapy has been described as a factor significantly influencing the development of fat necrosis (18).

\section{Objectives}

Aim of this study was to compare the incidence and mammographic appearance of fat necrosis in the postsurgical breast on digital mammograms with and without adjuvant treatment. This will serve as a comparison study to the upcoming neoadjuvant radiotherapy (neoRT) trial (19), where radiation therapy will be administered prior to surgical therapy.

\section{Patients and Methods}

\subsection{Patient Cohort}

This study was registered as a service evaluation at Cambridge University Hospitals NHS Foundation Trust to evaluate if the rate of detected or caused fat necrosis is higher than expected. A retrospective analysis of all consecutive patients with benign findings managed by surgical excision between January 2011 and December 2014 was performed. Forty-seven patients diagnosed with a lesion of uncertain malignant potential on needle biopsy required open surgical biopsy and confirmed to be non-malignant. Eight patients were excluded as no post-surgical mammographic follow-up was available, leaving a total of 39 patients in the study group.

Also, an analysis of all cancer patients diagnosed in year 2013, the mid point of the benign surgical cohort, in the Cambridge screening programme was performed. Primary surgery was performed in 128 patients with screendetected breast cancers. Four patients were excluded as no mammography follow-up was available. Another 43 patients were excluded as mastectomy was performed. Hence, 81 patients built the study cohort with surgical excision of a malignant finding.

The cohorts were analyzed regarding age at the time of surgery, lesion size and additional adjuvant therapy. Available mammograms as follow-up tests were noted and analyzed regarding signs of fat necrosis.

\subsection{Image Analysis}

One reader with six years of experience in reading digital mammography (H.P.) performed the image analysis. The reader was aware of the laterality of the finding as well as the pre-therapeutic imaging but was not aware of the treatment additional to surgical excision. It was noted if there was any sign of (developing) fat necrosis on postsurgical imaging, such as lucencies, masses or calcifications. Measurement of the findings was performed. Findings were noted separately for each imaging time point. The different appearances of fat necrosis were noted.

\subsection{Statistical Analysis}

Statistical analysis was performed using SPSS (SPSS Inc. Released 2007. SPSS for Windows, version 16.0. Chicago, Ill., USA). Results are given as mean \pm standard deviation (SD). Student's $t$-test was used in case of continuous data, $\chi^{2}$-test in case of categorical data. Logistic regression was performed. Z-transform was used for age and lesion size ([x-mean]/standard deviation). P values $\leq 0.05$ were considered statistically significant.

\section{Results}

\subsection{Patient Characteristics}

Mean patient age at the time of surgery was $54.6 \pm 6.5$ years in the benign and $59.8 \pm 7.8$ years in the malignant group $(\mathrm{P}=0.0002)$.

\subsection{Lesion Characteristics}

In the benign group, the mean pathological size of the lesion demanding excision was $15.3 \mathrm{~mm}$ overall. The mean lesion size was $16.1 \mathrm{~mm}$ on mammography, and $17.5 \mathrm{~mm}$ on ultrasound. Thirty-two of 39 lesions (82.1\%) were visible on mammography; whereas, 15/39 lesions (38.5\%) were visible on ultrasound. Of the 32 lesions visible on mammography, $59.4 \%$ were calcifications only, followed by lucency and calcifications (34.4\%).

In the malignant group, the mean whole pathological tumour size was $16.1 \mathrm{~mm}$. The mean lesion size did not differ between the groups $(\mathrm{P}=0.735)$. The mean diameter of invasive cancer was $12.7 \mathrm{~mm}$ and $19.7 \mathrm{~mm}$ of pure noninvasive tumor. Of all malignant tumors 74.1\% (60/81) were invasive, 21 (35.0\%) of them with an extratumoural noninvasive component. Histopathology showed carcinomas of no special type (NST) in $85.0 \%$ (51/60), invasive lobular carcinomas (ILC) in 8.3\% (5/60), and mucinous and mixed NST/ILC in 3.3\% (2/60) each. Invasive cancers were grade 1 in $28.3 \%$ (17/60), grade 2 in $46.7 \%$ (28/60), grade 3 in $21.7 \%$ (13/60), and the grade was unknown in 3.3\% (2/60) cases. Pure non-invasive cancers made up 25.9\% (21/81), of which 
17 were ductal carcinoma in situ (DCIS) (81.0\%) and four atypical lobular neoplasias (ALN; 19.0\%).

\subsection{Surgical Therapy}

In the benign group, surgical excision was performed with help of wire marking. The lesions were impalpable in 14 cases (35.9\%). Repeated surgery was performed in one case $(2.6 \%)$ due to suspicion of microinvasion at the margin of atypical ductal hyperplasia (ADH) after the first surgery with no residual disease in the second surgical specimen and therefore, final diagnosis of ADH.

In the malignant group, breast conserving therapy was performed in 18 cases $(22.2 \%)$ and breast conserving therapy with additional axillary surgery in 63 cases (77.8\%). Repeated surgery was performed in 17 cases (21.0\%), 15 cases of them $(88.2 \%)$ to obtain tumor-free margins and two cases (11.8\%) to complete surgical axillary therapy.

\subsection{Adjuvant Therapy in the Malignant Group}

Whole breast radiation therapy was performed in 75 patients (75/81; 92.6\%). No breast irradiation was performed in six patients with in situ carcinoma. In 16 patients $(16 / 75 ; 21.3 \%)$, chemotherapy in addition to radiotherapy was performed sequentially. Forty-six patients (56.8\%) received adjuvant hormonal treatment in addition to radiotherapy (estrogen receptor modulator or aromatase inhibitor). Hormonal treatment without radiation or chemotherapy was performed in one case $(1 / 46 ; 2.2 \%)$. The combination of breast irradiation and hormonal treatment was applied in 36 cases (78.3\%) and in nine cases (19.6\%), radiation, chemotherapy and hormonal treatment were performed.

\subsection{Mammography Follow-Up}

Mean available mammography follow-up was 4.1 years in the benign and 3.6 years in the malignant group. Bilateral mammograms in both views were available four times after surgery in four patients, three times in nine patients, two times in 16 patients and only one follow-up was available in 10 patients in the benign group. Bilateral mammograms in both views were available four times after surgery in 51 patients, three times in 25 patients, two times in two patients and only one follow-up was available in three patients in the malignant group.

\subsection{Fat Necrosis}

A development of fat necrosis on mammography follow-up was observed in $12.8 \%$ (5/39) of the patients in the benign group and in $32.1 \%$ (26/81) of the patients in the malignant group $(\mathrm{P}=0.024)$ (Table 1$)$. Logistic regression of all
120 patients showed that the probability of fat necrosis development was significantly higher in case of a malignant compared to a benign lesion (odds ratio [OR]:3.57; 95\% confidence interval $[\mathrm{CI}]: 1.15$ - 11.01; $\mathrm{P}=0.027)$; whereas, patient age and lesion size did not significantly influence fat necrosis development $(\mathrm{P}=0.842$ and $\mathrm{P}=0.300$, respectively).

In one patient fat necrosis was detected as lucency only (Figure 1), in 18 as pure calcification, and in 11 patients as lucency with calcifications (Figure $2 \mathrm{~A}$ and $\mathrm{B}$ ). In one case a mass on post-surgical mammograms was aspirated and turned out to be an oil cyst (Figure 3). In one case without signs of fat necrosis, DCIS was detected on the second mammography follow-up in the benign group.

One of six patients $(16.7 \%)$ not treated with radiotherapy developed fat necrosis in the analyzed follow-up. With radiation therapy \pm hormonal treatment, 24 of 73 patients $(32.9 \%)$ developed fat necrosis, whereas four of 16 patients $(25.0 \%)$ treated with radiation and chemotherapy showed a development of fat necrosis. None of the nine patients treated with irradiation, chemotherapy and hormonal treatment showed fat necrosis development (Table 2 ). In the malignant group, logistic regression showed that hormonal treatment significantly influenced the development of fat necrosis, in detail lowering its chance as the odds ratio was < 1 (OR: 0.23; 95\% CI: 0.06 - 0.86; $\mathrm{P}=0.029$ ) (Table 3).

The frequency of fat necrosis development did not differ significantly between invasive and non-invasive cancers $(\mathrm{P}=0.83)$ (Table 2$)$.

Mean follow-up time until the first sign of fat necrosis was 2.6 years in the benign and 2.0 years in the malignant group $(\mathrm{P}=0.187)$. In the malignant group fat necrosis was first detected after more than 2 years in 10 cases (38.5\%) and in two cases (7.7\%) after more than 3 years.

Overall, the mean size of fat necrosis was $9.04 \mathrm{~mm}$ in the malignant group. Lucencies with calcifications measured $18.6 \mathrm{~mm}$ on average, pure calcifications $4.1 \mathrm{~mm}$. One case was a $12 \mathrm{~mm}$ measuring lucency only. In the benign group, fat necrosis calcifications had a mean diameter of $2.7 \mathrm{~mm}$, the mass measured $21 \mathrm{~mm}$ and there was one case with scattered fat necrosis calcifications in the periareolar scar tissue.

\section{Discussion}

Our study showed that significantly more cases of fat necrosis are observed in the operated breast when additional adjuvant treatment was performed compared to surgery alone ( $32.1 \%$ vs. $12.8 \%$ ). The chance of fat necrosis development was 3.5 times higher in case of a malignant lesion that was operated. In prior studies, radiation therapy 


\begin{tabular}{|c|c|c|c|c|c|}
\hline & Lucency only & Lucency and calcifications & Calcifications only & Mass & Total \\
\hline Benign & & & $4(80.0)$ & $1(20.0)$ & 5 \\
\hline Malignant & $1(3.8)$ & $11(42.3)$ & $14(53.8)$ & & 26 \\
\hline Total & $1(3.2)$ & $11(35.5)$ & $18(58.1)$ & $1(3.2)$ & 31 \\
\hline
\end{tabular}

${ }^{\mathrm{a}}$ Values are expressed as No. (\%).
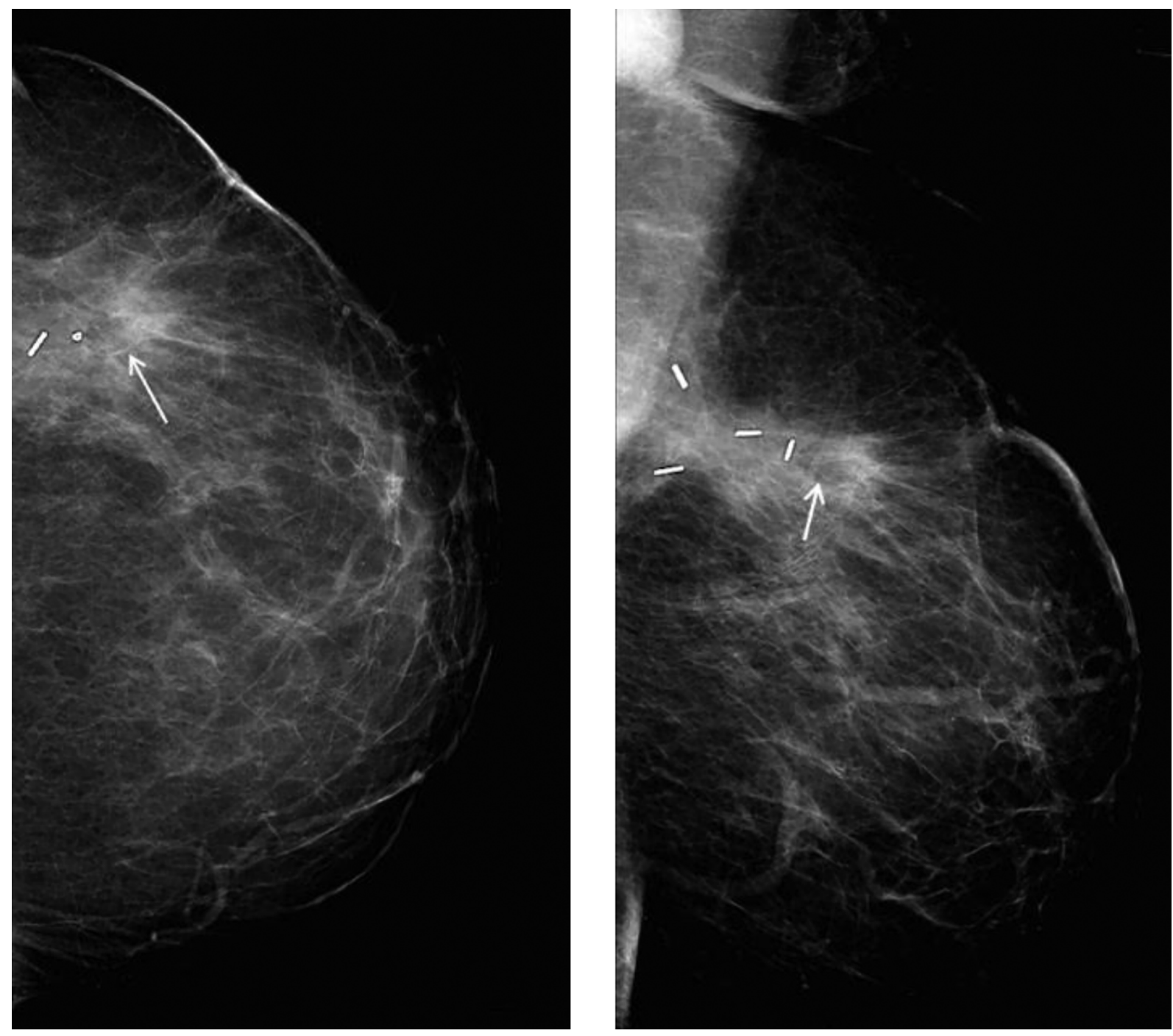

Figure 1. First post-surgical follow-up mammogram after breast conserving therapy in a 67-year-old patient due to $31 \mathrm{~mm}$ unifocal ductal carcinoma in situ (DCIS) in the left breast. Fat necrosis presented as a lucency anterior to the surgical clips (arrows).

was quoted as a factor significantly influencing the development of fat necrosis $(13,15,16)$. In our patient collective radiation did not significantly influence fat necrosis development. Chemotherapy alone has been reported as a factor influencing the development of fat necrosis in breast reconstruction after mastectomy (1). As none of the patients in our study collective received chemotherapy without irradiation of the breast, this could only be analyzed in logistic regression. The only factor significantly influencing the development of fat necrosis was hormonal treat- 

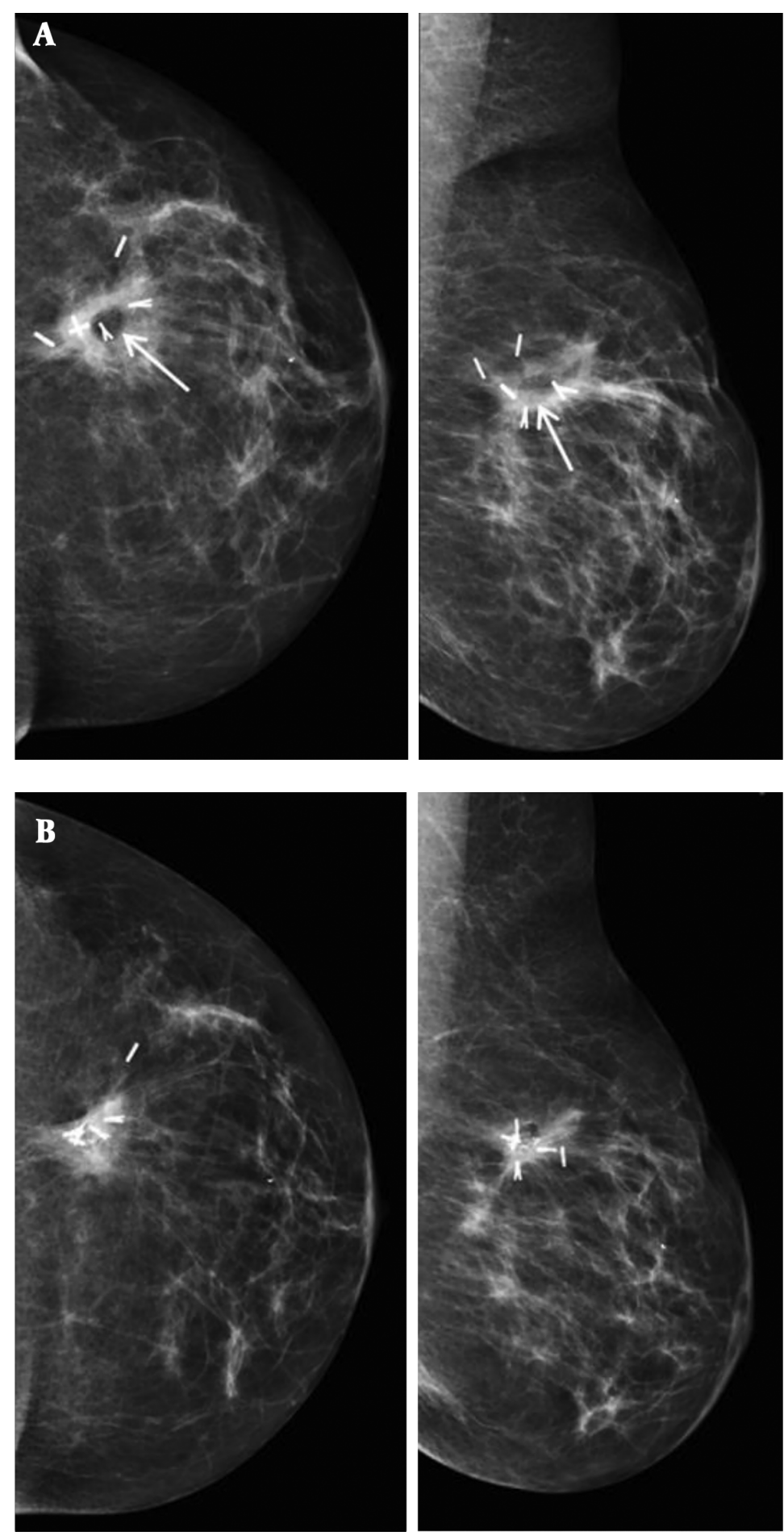

Figure 2. First (A) and forth (B) post-surgical follow-up mammogram after 0.9 and 4.0 years, respectively. Breast conserving therapy of a 5 mm invasive carcinoma in the left breast was performed. In the area of the post-surgical clips at the 12 o'clock position scarring tissue as well as a lucency in the centre of the clips is visible (A, arrows), which shows calcification later on (B) typical for fat necrosis. 

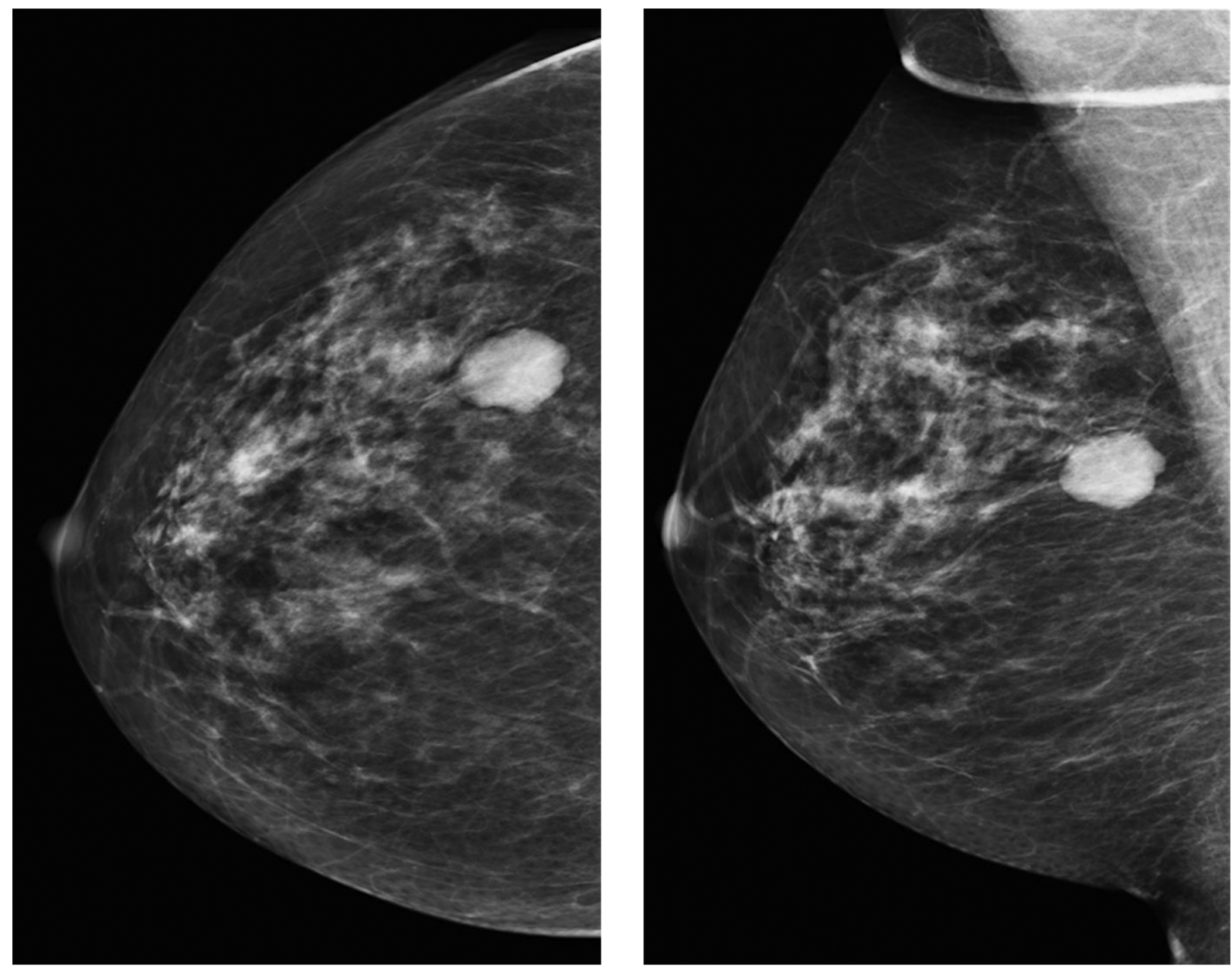

Figure 3. Second post-surgical mammogram of a 61-year-old patient 4 years after surgical excision of a B3 lesion in the right breast. There is a dense mass at the 9 o'clock position in the right breast with radiological benign appearance. The lesion showed cystic appearance on ultrasound and proved to be an oil cyst in aspiration.

\begin{tabular}{|c|c|c|c|c|c|c|c|}
\hline & no $\mathbf{R T}+\mid-\mathbf{H T}$ & RT only & $\mathbf{R T}+\mathbf{H T}$ & $\mathbf{R T}+\mathbf{C T}$ & $\mathbf{R T}+\mathbf{C T}+\mathbf{H T}$ & Total & Pvalue \\
\hline Fat necrosis present, invasive & & $4(17.4)$ & $11(30.6)$ & $4(57.1)$ & 0 & 19 & \\
\hline Fat necrosis absent, invasive & & $4(17.4)$ & $23(63.9)$ & $3(42.9)$ & $9(100)$ & 39 & \\
\hline Fat necrosis present, non-invasive & $1(16.7)$ & $5(21.7)$ & $1(2.8)$ & & & 7 & \\
\hline Fat necrosis absent, non-invasive & $5(83.3)$ & $10(43.5)$ & $1(2.8)$ & & & 16 & \\
\hline Total & 6 & 23 & 36 & 7 & 9 & 81 & 0.83 \\
\hline
\end{tabular}

Abbreviations: RT; radiotherapy, HT; hormonal therapy, CT; chemotherapy.

${ }^{\mathrm{a}}$ Values are expressed as No. (\%).

ment. In case of hormonal treatment, the chance of fat necrosis development was significantly lower.

The appearance of fat necrosis ranges from incidental benign findings to imaging features highly suggestive of malignancy $(20,21)$. The majority of fat necroses presented as pure calcifications in our study (59.4\%), followed by lucencies with calcifications (34.4\%). This is in accordance with literature pointing out dystrophic calcifications and radiolucent oil cysts as the most common mammographic findings (8). If fat necrosis becomes manifest as calcifica- 


\begin{tabular}{|c|c|c|c|c|}
\hline & OR & CI low ${ }^{a}$ & CI high $^{\mathrm{a}}$ & Pvalue \\
\hline Patient age ${ }^{b}$ & 0.959 & 0.565 & 1.628 & 0.877 \\
\hline Lesion size $^{b}$ & 2.106 & 0.972 & 4.562 & 0.059 \\
\hline Radiotherapy & 2.964 & 0.291 & 30.189 & 0.359 \\
\hline Chemotherapy & 0.339 & 0.079 & 1.446 & 0.144 \\
\hline Hormonal treatment & 0.231 & 0.062 & 0.860 & $0.029^{*}$ \\
\hline
\end{tabular}

Abbreviation: OR, odds ratio

${ }^{a} \mathrm{CI}$ high, upper limit of $95 \%$ confidence interval; CI low, lower limit of $95 \%$ confidence interval.

${ }^{\mathrm{b}} \mathrm{Z}$-transform was used.

tions only the probability that it is mistakenly scored as suspicious for recurrent malignancy is high. In case of a lucency in addition to the calcification, the fat necrosis can be verified on ultrasound (21). The mean follow-up time until fat necrosis manifested on the mammogram was 2.6 years in the benign and 2.0 years in the malignant group. As $46.2 \%$ of the fat necrosis after surgery of breast cancer were initially detected more than two years after the surgery, it is reasonable to have annual follow-up of at least 5 years to be able to detect those changes. Even on MRI a delayed development of enhancement in fat necrosis has been reported (22). If accessible, MRI has an excellent negative predictive value in case of uncertain mammographic lesions without enhancement, but if there is enhancement in a probable fat necrosis, biopsy has to be performed to exclude malignancy (22).

Dependent on adjuvant treatment the development of fat necrosis was observed in $17 \%$ of the patients not treated with radiotherapy, in $33 \%$ of the patients treated with radiation therapy \pm hormonal treatment and in $25 \%$ of the patients treated with radiation and chemotherapy in our study. The reported rate of fat necrosis after breast conserving therapy and irradiation is up to $40 \%$ and both radiation and chemotherapy have been shown to increase the risk of development of fat necrosis $(16,18)$. However, in our series, the effect of adjuvant chemotherapy did not add to the effect of radiotherapy. As fat necrosis often occurs around the tumour bed, it will be highly interesting to compare our results with the incidence, location, morphology and time of appearance of fat necrosis after neoadjuvant radiotherapy. As part of the neoRT trial, surgical excision of the malignant findings will be performed after neoadjuvant radiotherapy, so part of the radiated tissue will not stay in the breast. Perhaps this will end in a lower rate of fat necrosis, and the time of appearance after surgery will differ.

There are limitations in our study. First, due to the small case numbers overall and the small case numbers of histopathological cancer types other than no special type (NST), no analysis of a possible impact of tumor histopathology on the development of fat necrosis could be performed. Second, the presence of fat necrosis was only rated radiographically as ultrasound follow-up is not performed routinely in Cambridge or the UK.

In conclusion, this study shows the supposable impact of adjuvant therapy to the development of fat necrosis after surgery in a series of cancer patients with adjuvant radiotherapy compared to patients with surgery of benign findings. This has to be compared to the incidence, morphology, and time span of the potential development of fat necrosis after neoadjuvant radiotherapy with and without chemotherapy and hormonal treatment as part of the radiated tissue will be removed during surgery.

\section{Acknowledgments}

We gratefully acknowledge the help of Charlotte Coles, $\mathrm{MD}$, correcting the manuscript.

\section{Footnotes}

Authors' Contributions: Study concept and design: Matthew G Wallis. Analysis and interpretation of data: Heike Preibsch and Matthew G Wallis. Drafting of the manuscript: Heike Preibsch. Critical revision of the manuscript for important intellectual content: Matthew G Wallis. Statistical analysis: Heike Preibsch and Matthew G Wallis.

Conflict of Interests: There are no conflict of interests of any of the authors or institutions.

Funding/Support: No funding.

\section{References}

1. Lee BJ, Adair F. Traumatic fat necrosis of the female breast and its differentiation from carcinoma. Ann Surg. 1920;72(2):188-95. doi: 10.1097/00000658-192008000-00011. [PubMed: 17864327]. [PubMed Central: PMC1410519]. 
2. Pullyblank AM, Davies JD, Basten J, Rayter Z. Fat necrosis of the female breast-Hadfield re-visited. Breast. 2001;10(5):388-91. doi: 10.1054/brst.2000.0287. [PubMed: 14965612].

3. Kerridge WD, Kryvenko ON, Thompson A, Shah BA. Fat necrosis of the breast: A pictorial review of the mammographic, ultrasound, CT, and MRI findings with histopathologic correlation. Radiol Res Pract. 2015;2015:613139. doi: 10.1155/2015/613139. [PubMed: 25861475]. [PubMed Central: PMC4378709].

4. Bassett LW, Gold RH, Cove HC. Mammographic spectrum of traumatic fat necrosis: The fallibility of "pathognomonic" signs of carcinoma. AJR Am J Roentgenol. 1978;130(1):119-22. doi: 10.2214/ajr.130.1.119. [PubMed: 413396].

5. Chala LF, de Barros N, de Camargo Moraes P, Endo E, Kim SJ, Pincerato KM, et al. Fat necrosis of the breast: mammographic, sonographic, computed tomography, and magnetic resonance imaging findings. Curr Probl Diagn Radiol. 2004;33(3):106-26. doi: 10.1067/j.cpradiol.2004.01.001. [PubMed:15215818].

6. Hogge JP, Robinson RE, Magnant CM, Zuurbier RA. The mammographic spectrum of fat necrosis of the breast. Radiographics. 1995;15(6):1347-56. doi: 10.1148/radiographics.15.6.8577961. [PubMed: 8577961].

7. Bilgen IG, Ustun EE, Memis A. Fat necrosis of the breast: Clinical, mammographic and sonographic features. Eur J Radiol. 2001;39(2):92-9. doi: 10.1016/s0720-048x(00)00303-x. [PubMed: 11522417].

8. Tan PH, Lai LM, Carrington EV, Opaluwa AS, Ravikumar KH, Chetty N, et al. Fat necrosis of the breast-a review. Breast. 2006;15(3):313-8. doi: 10.1016/j.breast.2005.07.003. [PubMed:16198567].

9. Pui $\mathrm{MH}$, Movson IJ. Fatty tissue breast lesions. Clin Imaging. 2003;27(3):150-5. doi: 10.1016/S0899-7071(02)00536-3. [PubMed: 12727050].

10. Taboada JL, Stephens TW, Krishnamurthy S, Brandt KR, Whitman GJ. The many faces of fat necrosis in the breast. AJR Am I Roentgenol. 2009;192(3):815-25. doi: 10.2214/AJR.08.1250. [PubMed: 19234281].

11. Clarke D, Curtis JL, Martinez A, Fajardo L, Goffinet D. Fat necrosis of the breast simulating recurrent carcinoma after primary radiotherapy in the management of early stage breast carcinoma. Cancer. 1983;52(3):442-5. doi: 10.1002/1097-0142(19830801)52:3<442::aidcncr2820520310>3.0.co;2-w. [PubMed: 6861083].

12. Stigers KB, King JG, Davey DD, Stelling CB. Abnormalities of the breast caused by biopsy: Spectrum of mammographic findings. AJR Am J Roentgenol. 1991;156(2):287-91. doi: 10.2214/ajr.156.2.1898800.
[PubMed: 1898800].

13. Rebner M, Pennes DR, Adler DD, Helvie MA, Lichter AS. Breast microcalcifications after lumpectomy and radiation therapy. Radiology. 1989;170(3 Pt 1):691-3. doi: 10.1148/radiology.170.3.2492670. [PubMed: 2492670].

14. DiPiro PJ, Meyer JE, Frenna TH, Denison CM. Seat belt injuries of the breast: Findings on mammography and sonography. $A J R$ Am J Roentgenol. 1995;164(2):317-20. doi: 10.2214/ajr.164.2.7839961. [PubMed: 7839961].

15. Rostom AY, el-Sayed ME. Fat necrosis of the breast: An unusual complication of lumpectomy and radiotherapy in breast cancer. Clin Radiol. 1987;38(1):31. doi: 10.1016/s0009-9260(87)80394-x. [PubMed:3816064]

16. Ajkay N, Collett AE, Bloomquist EV, Gracely EJ, Frazier TG, Barrio AV. A comparison of complication rates in early-stage breast cancer patients treated with brachytherapy versus whole-breast irradiation. Ann Surg Oncol. 2015;22(4):1140-5. doi:10.1245/s10434-014-4131-6. [PubMed: 25319575].

17. Rakhra S, Bethke K, Strauss J, Hayes JP, Hansen N, Khan SA, et al Risk factors leading to complications in early-stage breast cancer following breast-conserving surgery and intraoperative radiotherapy. Ann Surg Oncol. 2017;24(5):1258-61. doi: 10.1245/s10434-016-5679-0. [PubMed: 27853900].

18. Li L, Chen Y, Chen J, Chen J, Yang B, Li J, et al. Adjuvant chemotherapy increases the prevalence of fat necrosis in immediate free abdominal flap breast reconstruction. J Plast Reconstr Aesthet Surg. 2014;67(4):4617. doi: 10.1016/j.bjps.2014.01.008. [PubMed: 24530060].

19. Lightowlers SV, Boersma LJ, Fourquet A, Kirova YM, Offersen $\mathrm{BV}$, Poortmans $\mathrm{P}$, et al. Preoperative breast radiation therapy: Indications and perspectives. Eur J Cancer. 2017;82:184-92. doi: 10.1016/j.ejca.2017.06.014. [PubMed: 28692950].

20. Evers K, Troupin RH. Lipid cyst: Classic and atypical appearances. AJR Am J Roentgenol. 1991;157(2):271-3. doi: 10.2214/ajr.157.2.1853804. [PubMed: 1853804].

21. Soo MS, Kornguth PJ, Hertzberg BS. Fat necrosis in the breast: Sonographic features. Radiology. 1998;206(1):261-9. doi: 10.1148/radiology.206.1.9423681. [PubMed: 9423681].

22. Solomon B, Orel S, Reynolds C, Schnall M. Delayed development of enhancement in fat necrosis after breast conservation therapy: A potential pitfall of MR imaging of the breast. AJR Am J Roentgenol. 1998;170(4):966-8. doi:10.2214/ajr.170.4.9530045. [PubMed: 9530045]. 